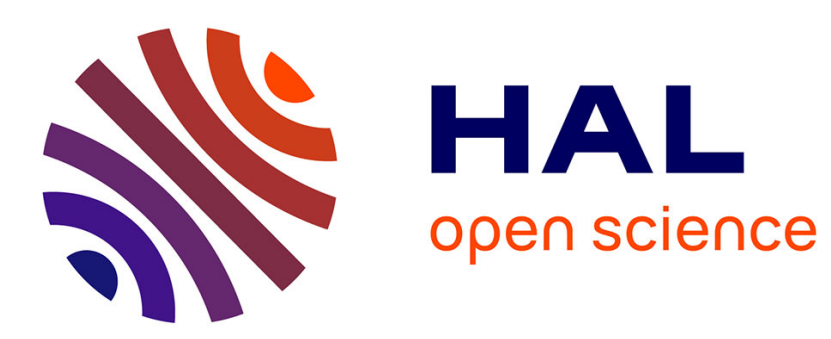

\title{
Utilisation de marqueurs pour la sauvegarde de la variabilité génétique des populations \\ C. Chevalet
}

\section{To cite this version:}

C. Chevalet. Utilisation de marqueurs pour la sauvegarde de la variabilité génétique des populations.

Productions Animales, 1992, hs (hs), pp.295-297. hal-00896035

\section{HAL Id: hal-00896035 \\ https://hal.science/hal-00896035}

Submitted on 1 Jan 1992

HAL is a multi-disciplinary open access archive for the deposit and dissemination of scientific research documents, whether they are published or not. The documents may come from teaching and research institutions in France or abroad, or from public or private research centers.
L'archive ouverte pluridisciplinaire HAL, est destinée au dépôt et à la diffusion de documents scientifiques de niveau recherche, publiés ou non, émanant des établissements d'enseignement et de recherche français ou étrangers, des laboratoires publics ou privés. 
C. CHEVALET

INRA Laboratoire de Génétique cellulaire BP 27

31326 Castanet-Tolosan Cedex
Apports actuels et futurs des

marqueurs génétiques dans

l'amélioration des populations

animales

Utilisation de marqueurs pour la sauvegarde de la variabilité génétique des populations

Résumé. On discute une possibilité d'utiliser des marqueurs polymorphes pour contrebalancer, dans une population d'effectif limité, les effets de la dérive aléatoire des fréquences alléliques. Le critère de sélection utilisé est un index favorisant le maintien de l'hétérozygotie aux locus marqueurs. Dans des populations d'effectif petit (moins de 100 reproducteurs efficaces) il semble qu'un marquage peu dense mais uniformément réparti (3 marqueurs pour 100 centimorgans) permette des gains sensibles pour le maintien de l'hétérozygotie sur l'ensemble du génome avec une intensité de sélection faible. Par ailleurs la réponse observée aux marqueurs s'avère très élevée même avec un assez grand nombre de marqueurs sélectionnés simultanément.

En l'absence de marquage génétique, la variabilité génétique est appréciée par l'analyse statistique de la variance de caractères quantitatifs. Contrôler cette variabilité consiste à estimer, au fil des générations, les variations des composantes de la variance, notamment de la variance génétique additive sur laquelle s'appuie la sélection.

Dans une population en conservation, ou une lignée témoin non soumise à sélection, sinon à la sélection naturelle, les méthodes de calcul de l'évolution de la consanguinité permettent de prédire l'évolution des composantes génétiques de la variance, selon les effectifs, la démographie de la population, les règles d'accouplements (Cockerham 1967, Choy et Weir 1978, Rochambeau et Chevalet 1985). Si des marqueurs polymorphes sont disponibles ils peuvent être introduits pour contrôler la dérive, et pour définir un protocole de gestion d'une population. On peut chercher par exemple à maintenir la variabilité génétique dans son état initial, en maintenant dans la population les allèles à leurs fréquences initiales.

Des essais théoriques préliminaires (Chevalet et Rochambeau 1986) suggèrent que cette approche peut prolonger la survie simultanée de plusieurs allèles en plusieurs locus indépendants, en définissant un index favorisant la reproduction des individus porteurs d'allèles rares. Cette approche pose deux problèmes :

- d'une part il faut exercer une pression de sélection qui induit systématiquement une limitation du nombre des reproducteurs et donc un risque supplémentaire de dérive aléatoire ;

- d'autre part il faut évaluer l'impact d'une sélection effectuée en quelques marqueurs sur la structure globale du génome.

On suppose donc qu'on dispose d'un échantillon uniformément distribué de marqueurs : Peut-on les utiliser efficacement, selon leur densité et selon les intensités de sélection applicables, pour ralentir le processus de dérive génétique inévitable dans une population d'effectif limité ? Peut-on exercer un contrôle sur l'ensemble du génome à partir d'un nombre restreint de marqueurs?

\section{1 / Méthode}

\section{La population et le génome}

On considère une population d'effectif limité N, non structurée (un seul groupe de reproduction). Cet effectif correspond au nombre d'individus susceptibles de se reproduire, et conduirait sans sélection à une diminution de l'hétérozygotie moyenne $\mathrm{H}$ selon la formule :

$\mathrm{H}(\mathrm{g}+1)=(1-1 /(2 \mathrm{~N})) \mathrm{H}(\mathrm{g})$

où $\mathrm{H}(\mathrm{g})$ est l'hétérozygotie à la génération ( $\mathrm{g}$ )

On considère par ailleurs pour l'espèce considérée une carte génétique simplifiée, formée de $\mathrm{C}$ paires de chromosomes de même longueur, et sur chaque chromosome un même nombre de locus marqueurs bialléliques observables utilisés pour une sélection ; soit L le nombre total de marqueurs.

\section{Caractères étudiés et critère de sélection}

A chaque génération la population est décrite par son taux moyen d'hétérozygotie aux locus marqueurs, et par son taux moyen d'hétérozygotie global sur l'ensemble du génome.

Les individus reproducteurs sont sélectionnés parmi les $\mathrm{N}$ individus susceptibles de se reproduire, selon un index égal au taux d'hétérozygotie mesuré aux L locus marqueurs. 


\section{Méthode de calcul}

Le système est étudié par une simulation aléatoire du processus génétique. Chaque individu de la population est décrit par son génotype sur un ensemble de $\mathrm{T}$ locus répartis uniformément sur les chromosomes, chaque chromosome étant représenté par T/C locus avec des taux de recombinaison égaux entre deux locus adjacents. La méiose est simulée par un processus de Poisson le long du chromosome, correspondant à la fonction de carte de Haldane (sans interférence).

A chaque locus, deux allèles sont répartis avec des probabilités égales entre les individus fondateurs. Dans chaque répétition, la population est soumise à une évolution sans sélection pendant une vingtaine de générations avec l'effectif de $\mathrm{N}$ individus reproducteurs, de façon à générer des conditions initiales qui présentent des valeurs vraisemblables des déséquilibres de liaison dus au hasard. La population est ensuite soumise à une sélection pour le taux d'hétérozygotie aux locus marqueurs pendant 40 générations.

L'hétérozygotie globale est approchée par sa mesure sur les T locus tandis que la sélection porte sur le taux mesuré sur les L marqueurs.

Pour chaque série de paramètres, 100 répétitions ont été effectuées.

\section{2 / Résultats}

Les calculs ont été effectués pour les valeurs suivantes des paramètres :

$\mathrm{C}=10$ paires de chromosomes de $100 \mathrm{cM}$ de longueur ;

$\mathrm{T}=110$ locus ( $10 \mathrm{cM}$ entre locus adjacents);

$\mathrm{L}=20$ ( 2 marqueurs par chromosome ),

ou 30 ( 3 marqueurs par chromosome);

$\mathrm{N}=25,50$ ou 100 ;

Taux de sélection (proportion s des individus adultes retenus pour la reproduction):

$\mathrm{s}=0,50$ à 1,00 .

Les résultats concernent les taux d'hétérozygotie globale (figures 1 à 3). Les gains réalisés par la sélection sont exprimés en pourcentage par rapport aux valeurs attendues sans sélection, après 20 ou 40 générations, pour les 3 valeurs considérées du nombre total d'individus disponibles, et pour les 2 densités de marqueurs.

\section{3 / Discussion}

Ces résultats préliminaires montrent une influence de la densité des marqueurs sur l'efficacité de la méthode envisagée. Avec 2 marqueurs par chromosome, la diminution de la dérive aléatoire globale est faible sur l'ensemble du génome alors que la méthode de sélection semble très efficace sur les marqueurs contrôlés, peu liés. Le gain global est faible et une sélection assez intense induit rapidement un effet accru de la dérive, caractérisé par des valeurs inférieures à celle obtenue sans sélection. Avec 3 marqueurs par chromosome uniformément répartis sur une centaine de centiMorgans, l'efficacité pourrait devenir non négligeable, à condition que la pression de sélection demeure faible. On peut noter l'existence de valeurs optimales faibles de l'intensité de sélection.
Figure 1. Gains relatifs sur le taux d'hétérozygotie, dus à la sélection sur un échantillon de marqueurs $(N=25)$.

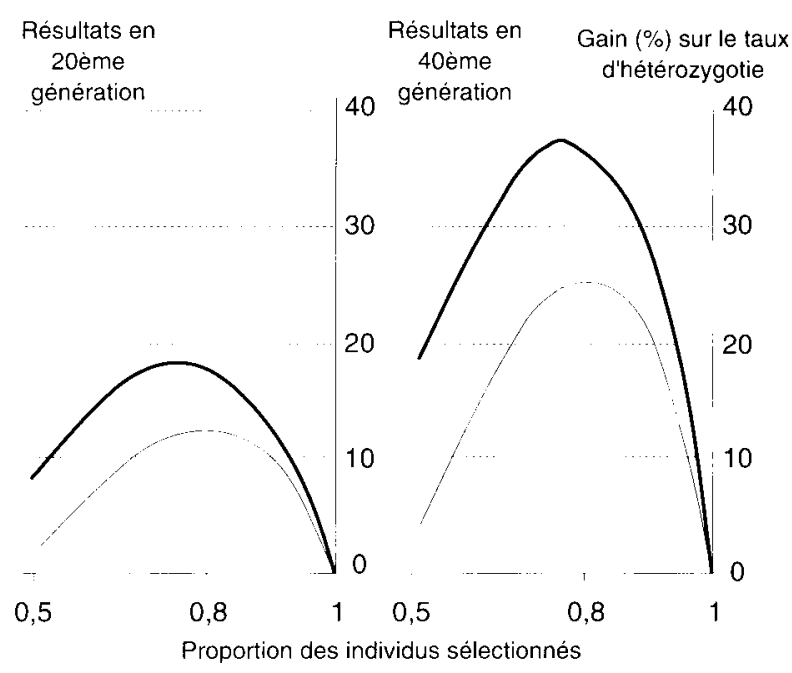

Contrôle exercé sur 3 marqueurs par chromosome Contrôle exercé sur 2 marqueurs par chromosome

Figure 2. Gains relatifs sur le taux d'hétérozygotie, dus à la sélection sur un échantillon de marqueurs $(N=50)$.

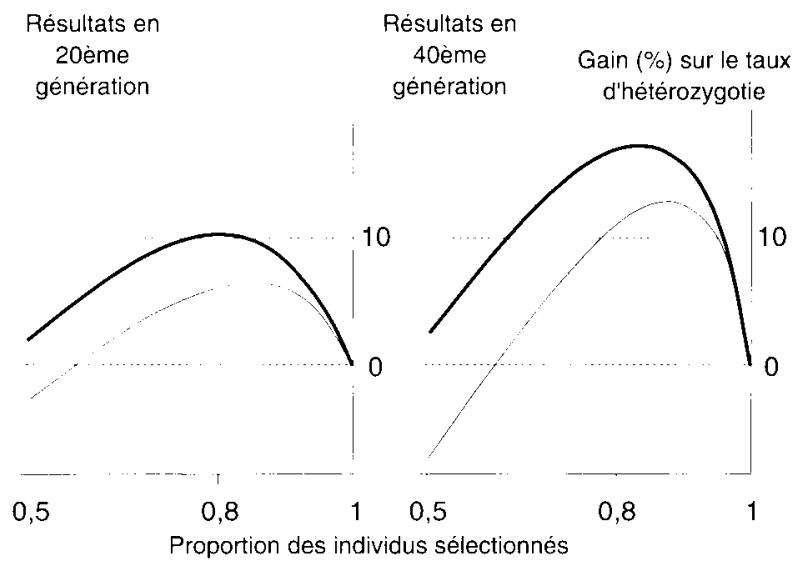

Contrôle exercé sur 3 marqueurs par chromosome Contrôle exercé sur 2 marqueurs par chromosome

Figure 3. Gains relatifs sur le taux d'hétérozygotie, dus à la sélection sur un échantillon de marqueurs $(N=100)$.

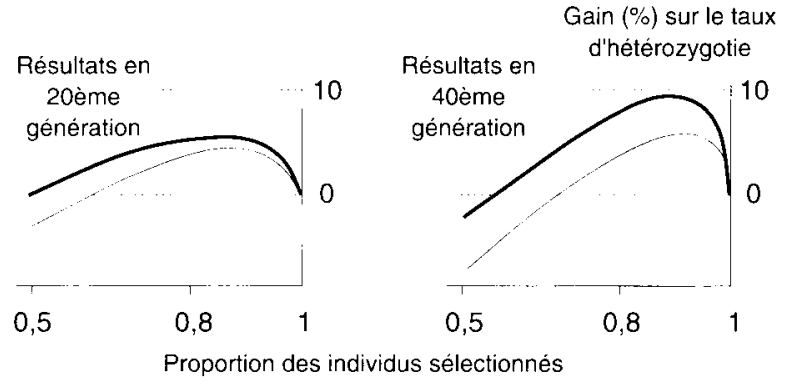

Contrôle exercé sur 3 marqueurs par chromosome

Contrôle exercé sur 2 marqueurs par chromosome 
Il apparait sur les figures que l'efficacité relative est d'autant meilleure que l'effectif de la population est plus faible. Cette différence doit être en partie attribuée aux conditions initiales différentes. Dans les différents cas une phase d'évolution préalable sans sélection a été simulée, pendant 20 générations, pour chacun des effectifs $(25,50$, et 100 reproducteurs). Ce même délai induit des déséquilibres de liaison aléatoires d'autant plus importants en valeur absolue que l'effectif est plus faible, à nombre de générations égal (Weir et Cockerham 1974). Les effets d'entrainement qui permettent de contrôler l'ensemble du génome à partir des marqueurs sont donc d'autant plus forts que la population est de taille plus restreinte. Selon ce principe, les résultats obtenus en 40ème génération, avec $\mathrm{N}=100$, et après une phase d'évolution sans sélection de 40 générations devraient être analogues à ceux obtenus en 20ème génération pour $\mathrm{N}=50$, après 20 générations sans sélection (figure 2 ) : cette prédiction est vérifiée pour des taux de sélection relativement forts (proportion sélectionnée comprise entre 50 et $80 \%$ ), ainsi que pour la valeur de l'optimum de sélection, mais l'accroissement de la taille de la population rend plus efficace des intensités de sélection plus faibles (résultats non présentés). Sauf pour de très petites tailles de la population $(\mathrm{N}=25)$, l'intensité de sélection optimale est voisine de $85 \%$, avec une variation faible des résultats entre 80 et $90 \%$, et d'autant plus faible que la taille de la population est plus grande.
Une autre façon de mesurer le gain obtenu est de comparer les nombres de générations correspondant, avec ou sans sélection, à une même perte de variabilité génétique. Ainsi, avec $\mathrm{N}=50$ et $\mathrm{Ns}=42$ (cas optimal), la sélection permet de maintenir jusqu'à la 15ème génération le niveau attendu sans sélection après 10 générations, ou jusqu'à la 40ème le niveau attendu après 25 .

L'efficacité de la sélection sur les régions marquées est en revanche très grande, malgré le nombre de marqueurs considérés simultanément (20 ou 30). Sur l'ensemble de ces marqueurs, et pour des proportions d'individus sélectionnés de 50 à $80 \%$, le taux d'hétérozygotie atteint entre les 20 ème et 40 ème générations est de l'ordre de 0,36 à 0,42 pour $\mathrm{N}=25$, de 0,475 à 0,485 pour $\mathrm{N}=50$, et supérieur à 0,495 pour $\mathrm{N}=100$, c'est à dire des valeurs proches du maximum de 0,50 , et qui se maintiennent très longtemps malgré la dérive. Cette efficacité locale du contrôle permet d'envisager un contrôle spécifique de quelques segments chromosomiques portant des gènes importants pour des caractères de production.

L'investigation de conditions plus générales est nécessaire pour préciser l'intérêt de cette approche. L'influence de la densité du marquage mérite une étude plus détaillée, en considérant des stratégies combinant progressivement plusieurs ensembles de marqueurs. La combinaison d'un contrôle assisté par marqueurs avec les méthodes de gestion par familles devrait aussi être abordée.

\section{Références bibliographiques}

Chevalet C. et de Rochambeau H., 1986. Variabilité génétique et contrôle des souches non consanguines. Sci. Tech. Anim. Lab., 11 : 251-257.

Choy S.C. and Weir B.S, 1978. Exact inbreeding coefficients in populations with overlapping generations. Genetics, 89 : 591-614.

Cockerham C.C., 1967. Group inbreeding and coancestry. Genetics, $56: 89-104$.
Rochambeau H. de, et Chevalet C., 1985. Minimisation des coefficients de consanguinité dans les petites populations d'animaux domestiques. Génét. Sél. Evol., 17 : 459-480.

Weir B.S. and Cockerham C.C., 1974. Behavior of pairs of loci in finite monoecious populations. Theor. Pop. Biol., 6 : 323-354. 